\title{
PREDICTION OF GRAZING GRASSLAND PRODUCTIVITY BY LOGISTIC TYPE MODELS
}

\author{
Masae SHIYOMI, Nobuaki KOYAMA and Mikinori TSUIKI
}

National Grassland Research Institute, Ecology

Division, Nishinasuno, Tochigi 329-27, Japan

\begin{abstract}
SUMMARY
Ecosystem models to analyse energy and matter flows in Japanese grazing grassland have been developed during the past 10 years. These models have contributed much to ecosystem studies but they are not suitable, from the farmers' viewpoint, for predicting grassland productivity for two reasons: (1) as they have generally large and complex structures, they do not fit individual farmers' small personal computers; and (2) they contain so many variables that farmers cannot measure precise values of all of them in their fields.

In this paper, we propose six small simple population models for describing plant and animal growth which can be used by farmers, compare these with an ecosystem model, and show that such population models are suitable for predicting short term grassland productivity. In the population models, plants grow on the basis of logistic theory, and the animal population ingests the necessary nutrients from grassland and consume its energy in proportion to the 3/4 power of its total body weight. The only initial value to be measured, which is necessary for predicting grazing grassland productivity, is aboveground plant biomass, and in some cases animal body weight grazed. Moreover, if a proper technique for automatically monitoring aboveground plant biomass can be developed, a combination of population model and monitoring system will contribute much to prediction and management of grassland productivity.
\end{abstract}

KEY WORDS: Grazing grassland, Prediction of grassland productivity, Logistic type models.

\section{INTRODUCTION}

A grazing grassland forms an ecosystem which is composed of elements such as soil microorganisms, plants, animals and the natural environments affecting living organisms in grassland. Human beings have given various agricultural impetuses to the ecosystem and have harvested animal products as protein sources from the ecosystem for ages. In the past 10 years, attempts to convert ecosystem structure into mathematical structure, $\underline{i} \cdot$.e.., ecosystem model, have been made, $\underline{e} . \mathrm{g} .$, as SHIYOMI et al.(1983); in these attempts various production conditions which could never be realized in an actual farmer's field have been actualized on a computer. Such ecological modelling bring immediate effects on ecosystem studies, but they are not necessarily useful for individual farmers, because farmers can use small computers but not large computers, and furthermore they do not like to work with the many precise variables which ecosystem models contain.

We will show in this paper that small and simple population models, as well as large and complex ecosystem models, can describe plant and animal growth, and that such population models are more useful for actual field in agricultural production. Similar subjects are treated by FICK and ONSTAD (1981) and WISIOL(1981).

\section{MODELS}

Let us arrange models, describing plant and animal growth in grazing grassland, in order of simplest to more complex as follows.

Model 1 $1^{\circ}$ : Let us assume that the plant growth follows a logistic theory and no consumption by animals occurs. Putting the plant biomass at time $t, x$, the growth rate, $r$ (constant) and the carring capacity, $K$ (constant, we obtain: 
$\mathrm{dx} / \mathrm{dt}=\mathrm{rx}(1-\mathrm{x} / \mathrm{K})$.

Model $2^{0}$ : Suppose that in the grassland described by (1) animals feed a ( constan) during a time period dt. Then we have:

$d x / d t=r x(1-x / K)-a$.

Model $3^{0}$ : This is a realisation of variable $r$. Usually $r$ takes high

values in spring but low values in summer and winter in Japan. In such a case, we have:

$d x / d t=r(t) x(1-x / K)-a$

where $r(t)$ is a function of $t$, and the seasonal changes are shown in Figure 1. Model $4^{\circ}$ : Suppose that animals are rotated among several paddocks. In

such a case, a is no longer a constant. Then we have: $d x / d t=r(t) x(1-x / K)-a(t)$.

Here, if there are animals in a given paddock $a(t)$ takes a constant positive value, but if there are no animals in the paddock $a(t)$ takes a zero value. Model $5^{\circ}$ : In the previous four models we consider only plant growth and consumption by animals. In the next step, let us take animal growth into consideration. Suppose that y indicates the animal biomass at time $t$, then, assuming continuous grazing, we have:

$d x / d t=r(t) x_{3}(1-x / K)-a(y)$ $\mathrm{dy} / \mathrm{dt}=\mathrm{cy}-\mathrm{ky} \mathrm{s} / \mathrm{4}$,

where $a(y)$ is a function of animal biomass, $y$, and $c$ is a constant for forage digestibility and utilization of energy ingested and $\mathrm{k}$ is a constant for energy consumption and excretion of animals. The second term in the second equation in (5) indicates that the energy consumed by an animal is in proportion to the $3 / 4$ power of the animal body weight.

Model $6^{\circ}$ : Assuming a rotational grazing in (5), the term of plant consumption by animals, a becomes a function of $y$ and $t$ :

$d x / d t=r(t) x_{3}(1-x / K)-a(y, t)$

where $a(y, t)$ is positive if there are animals in a given paddock, and $a(y, t)$ is zero otherwise.

Model 7 $7^{\circ}$ : The following grassland ecosystem model contains seven variables such as available aboveground plant portions $\left(x_{1}\right)$, unavailable portions $\left(x_{2}\right)$, belowground plant portions $\left(\mathrm{x}_{3}\right)$, plant standing dead material $\left(\mathrm{x}_{4}\right)$, soil surface plant litter $\left(\mathrm{x}_{5}\right)$, animal biomass $\left(\mathrm{x}_{6}\right)$ and excreta $\left(\mathrm{x}_{7}\right)$, and their reactions to meteorological and edaphic conditions:

$$
\mathrm{d}\left[\begin{array}{c}
x_{1} \\
x_{2} \\
\vdots \\
x_{7}
\end{array}\right]=\left[\begin{array}{llll}
f_{11} & f_{12} & \cdots & f_{17} \\
f_{21} & f_{22} & \cdots & f_{27} \\
\vdots & \vdots & & \vdots \\
f_{71} & f_{72} & \cdots & f_{77}
\end{array}\right]\left[\begin{array}{c}
x_{1} \\
x_{2} \\
\vdots \\
x_{7}
\end{array}\right] d t
$$

where $f_{i j}$ 's are function of $t$, meteorological conditions and edaphic and nutriental conditions. In this model, we can also easily express both rotational and continuous grazing by changing some of $f_{i j}$ 's. Refer to SHIYOMI et al.
(1983) for detailed information.

\section{CONFIGURATION OF THE MODELS AND DISCUSSION}

Some examples of models described previously are shown in Figure 1. In these examples, not only plant and animal growth but changes in the plant growth rate, $r$, with time are shown.

While it is certainly possible to predict plant and animal growth by using the grassland ecosystem model developed by SHIYOMI et al.(1983) because this model can mimic actual grazing grasslands, this ecosystem model contains many variables, so it is far from easy for farmers to use. The following two characteristics of our population models should suffice to make it usable by individual farmers or farmers' cooperatives: (1) they are small and simple, and anyone who wants to use it can compute predicted values using a small personal computer, and (2) farmers can easily measure an initial variable value necessary 

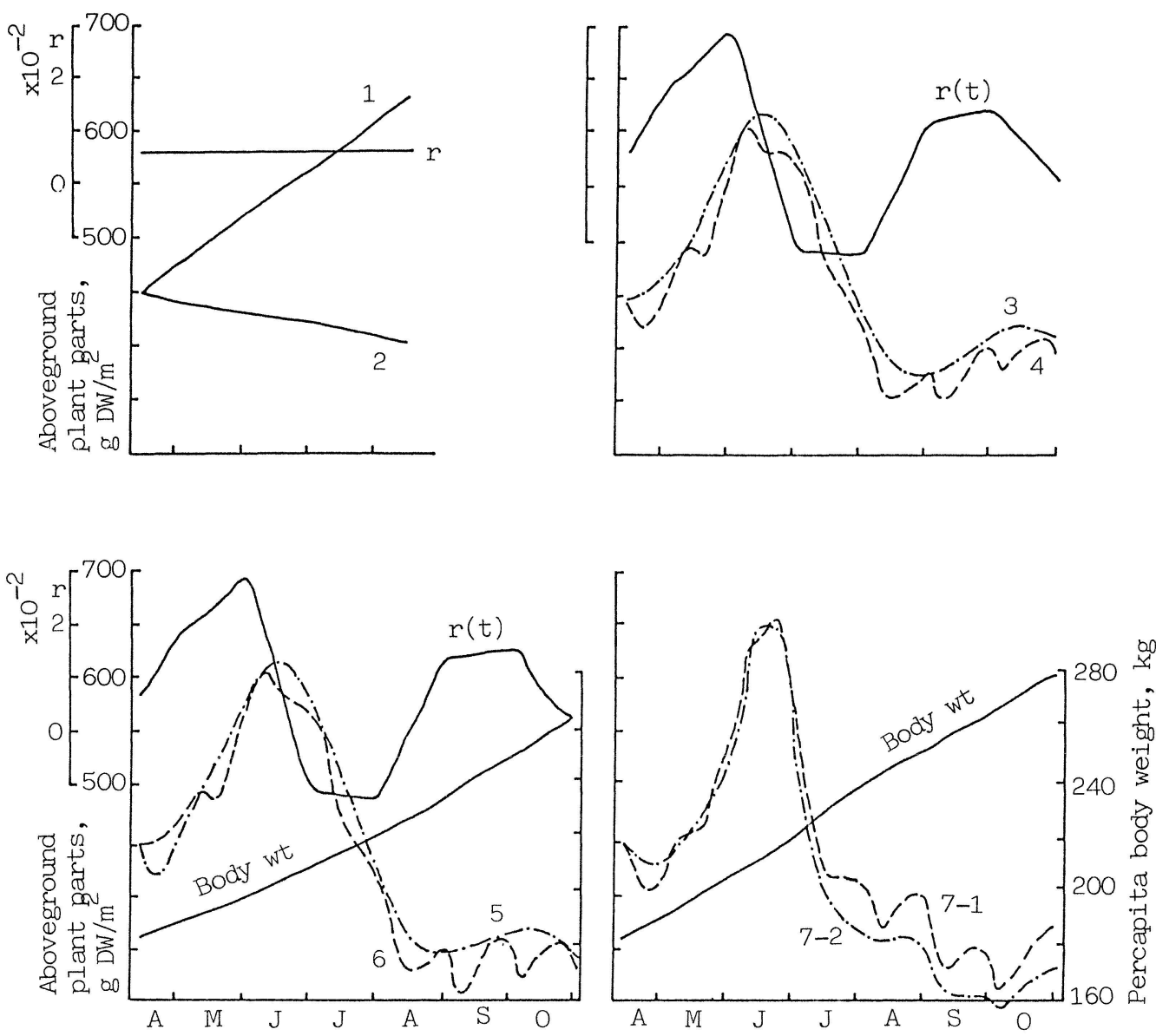

Figure 1. Simulated results of Model $1^{\circ}$ to $7^{\circ}$.

Nos. 1 to 3, 5 and 7-1 are results simulated under a continuous grazing and Nos. 4, 6 and 7-2 are those under a rotational grazing. Values of $r$ and $r(t)$ indicate the plant growth rate changes corresponding to plant biomass changes in each section of the 4 figures. The parameter $r$ takes a fixed value, 0.0063 , and $r(t)$ was calculated from the crop growth rate of plants measured at a grassland of the National Grassland Research Institute, which composes of plant species as orchard grass, tall fescue, red top, etc.. K is fixed on a value of $950 \mathrm{~g}$ dry weight $/ \mathrm{m}^{2}$. 1: Model 1 $1^{\circ}$; : Model $2^{\circ}$ for $a=1.855 \mathrm{~g}$ dry weight $/ \mathrm{m}^{2} ; 3$ : Model $3^{\mathrm{O}}$ for $a=1.855 \mathrm{~g}$ dry weight $/ \mathrm{m}^{\frac{z}{\tau}} ; 4$ : Model $4^{\circ}$ for $a(t)=7.420 \mathrm{~g}$ dry weight $/ \mathrm{m}^{2}$ when animals exist in a given paddock and $a(t)=0 \mathrm{~g}$ otherwise; 5 : Model $5^{\circ}, a(y)$ changes with the amount of grazing animals, and $\mathrm{c}$ and $\mathrm{k}$ are constants (see text); 6: Model $6^{\circ}, a(y, t)$ changes with the amount of grazing animals and the rotation schedule. Parameters $\mathrm{c}$ and $\mathrm{k}$ are the same as in Model $5^{\circ}$; 7: Model $\underline{7}^{\circ}, \underline{7-1}$ indicates a rotational grazing and $\underline{7-2}$ continuous grazing.

to calculate predictions. Personal computers are available for many farmers in Japan, and measuring aboveground plant biomass may be possible with them, though measuring belowground plant parts and soil surface litter may not. For this purpose, the population models $1^{0}$ to $\underline{6}^{\mathrm{O}}$ are useful, even the simplest, Model $1^{\mathrm{O}}$, for short term prediction. It is obvious from Figure 1 that the 
population models can approximate the aboveground biomass changes calculated from Model $\underline{7}^{\circ}$. If we have a prediction for a colder spring or a hotter summer than usual, it is also possible to diminish the plant growth in calculation by decreasing $r$-value.

In addition to development in model making, it is also necessary to develop an instrument for monitoring aboveground plant biomass automatically in an actual grassland. For example, if monitoring instruments for measuring plant biomass and meteorological data are installed, say, at 10 locations in a grassland area in Tochigi Prefecture and information is sent to some Information Center of Farmers' Cooperatives every moment, a proper decision for grassland managements can be made by predictions calculated throgh a population model from the information obtained at every location. Such large-area prediction of grassland productivity is an important concern for near future.

\section{LITERATURE CITED}

FICK, G.W. and D. ONSTAD(1981): Simple computer simulation models for forage management applications. Proc. XIV Intl Grassl. Cong.:483-485, Westview Press, Boulder, Colorado.

SHIYOMI, M., S. TAKAHASHI, T. AKIYAMA and T. HAKAMATA(1983): A system model for short and long term prediction of grazing pasture productivity. Bull. Natl Grassl. Res. Inst. 26:17-29.

WISIOL, K.(1981) Can simple model predict range yield? Summaries of Papers in XIV Intl Grassl. Cong.:257, Univ. of Kentucky, Lexington, Kentucky .

Part of this work is supported by Green Energy Project. 